\title{
Construct Validity of the Frenchay Activities Index for Community-Dwelling Elderly in Japan
}

\author{
Chang-Wan Han, ${ }^{1,2}$ Yuki YaJima, ${ }^{3}$ KaZuo NaKaJima, ${ }^{4}$ Eun-Joo Lee, ${ }^{2}$ \\ MaKiKo Meguro 5 and Masahiro KohzuKi ${ }^{1}$ \\ ${ }^{1}$ Department of Internal Medicine and Rehabilitation Science, Tohoku \\ University Graduate School of Medicine, Japan, \\ ${ }^{2}$ School of Health and Welfare, Woosong University, Deajeon, Korea, \\ ${ }^{3}$ Department of Public Health, Okayama University Graduate School of \\ Medicine and Dentistry, Japan, \\ ${ }^{4}$ Department of Health and Welfare Science, Okayama Prefectural University, \\ Japan, and \\ ${ }^{5}$ Division of Physiological Medicine, St. George's Hospital Medical School, \\ Cranmer Terrance, London, $U K$
}

Han, C.-W., Yajima, Y., Nakajima, K., Lee, E.-J., Meguro, M. and Kohzuki, M. Construct Validity of the Frenchay Activities Index for Community-Dwelling Elderly in Japan. Tohoku J. Exp. Med., 2006, 210 (2), 99-107 — Many researchers have developed various measures to evaluate the functional status of community-dwelling elderly. The Frenchay Activities Index (FAI) was developed to measure instrumental activities of daily living in stroke populations. The FAI has undergone the most intensive evaluation, but its factor structure has not been examined in Japan. The primary purpose of this study was to investigate the construct validity of the FAI for non-stroke community-dwelling elderly in Japan. The study subjects were 1,323 randomly selected community older residents in Hojo city, Ehime prefecture, the southern part of Japan. In order to investigate the construct validation, we performed factor analyses, i.e., exploratory factor analysis and confirmatory factor analysis. Furthermore, we examined the influences of age and gender on the FAI constructs. In the results, the FAI had a two-factor structure consisting of domestic chores, including washing clothes and preparing meals, and work and leisure, including gardening, driving and outings. Younger age was significantly related to increased performance on both factors. In addition, relative to females, males had a lower performance on the domestic chores and a greater performance on the work and leisure. These results suggest that the FAI satisfies the construct validity in the Japanese community-dwelling elderly. The FAI and its two factors provide important and clinically valuable information for understanding the patterns of functional status in the Japanese communitydwelling elderly. - Frenchay Activities Index; activities of daily living; disability; rehabilitation; elderly

(C) 2006 Tohoku University Medical Press

Received January 6, 2006; revision accepted for publication August 8, 2006.

Correspondence: Masahiro Kohzuki, M.D., Ph.D., Department of Internal Medicine and Rehabilitation Science, Tohoku University, Graduate School of Medicine, 1-1 Seiryo-machi, Aoba-ku, Sendai 980-8574, Japan. e-mail: kohzuki@mail.tains.tohoku.ac.jp 
The functional status is an essential factor determining the lifestyle and independence of older adults and has received considerable attention. Many researchers have developed various measures to evaluate the functional status and assess intervention effects for rehabilitation programs (Moskowitz and McCann 1957; Katz et al. 1963; Mahoney and Barthel 1965; Soening et al. 1965). A number of these were designed to evaluate the function in activities of daily living (ADL). Most of the ADL measures, for example, PULSES Profile (Moskowitz and McCann 1957), Katz ADL index (Katz et al. 1963), Kenny Self-Care Evaluation (Soening et al. 1965) and Barthel index (Mahoney and Barthel 1965), generally contain basic activities such as feeding, bathing and toilet use. However, such basic ADL measures are not necessarily adequate for independent-living in community older residents, which involves more complex activities such as housekeeping, recreation, hobbies, and social interaction (Whiting and Lincoln 1980).

In addition, it is well known that ADL scales generally show ceiling effects when applied to community-dwelling elderly (Nouri and Lincoln 1987). In order to overcome the weaknesses of these ADL scales, higher-order ADL assessments, such as the Instrumental Activities of Daily Livings (IADL) originally introduced by Lawton (Lawton and Brody 1969), have received increasing attention.

There have been many previous attempts to develop IADL assessment devices. Such measures include the Rivermead ADL scale (Whiting 1980), Nottingham Extended ADL scale (Nouri and Lincoln 1987), Hamrin Activity Index (Hamrin 1982) and Frenchay Activities Index (FAI) (Holbrook and Skilbeck 1983). A review of the psychometric investigations of these IADL scales revealed that the FAI had undergone the most intensive evaluation, especially concerning its construct validity (Khin-Heng 1995).

Construct validation studies on the FAI have examined group differences and the strength of the construct-related criteria (Wade et al. 1985). Another component of construct validation, factor structure, is well established. Factor structure is determined typically using a factor analysis. Previous factor analytic studies of the FAI have differed in terms of the extracted factor solution applied to a sample of stroke patients (Wade et al. 1985; Bond et al. 1992; Schuling et al. 1993; Sveen et al. 1999) and community dwelling older residents (Kburn et al. 1990; Bond et al. 1995), with the number of factors obtained from the results of the factor analyses ranging from two to four. Furthermore, the factor structure of the FAI has not been rigorously examined using a confirmatory factor analysis, although it is necessary to establish the factor structure. Ambiguity about the factor structure violates the assumption of the construct validity of the instrument.

In the present study, we addressed these concerns and aimed to specify the factor structure of the FAI using not only exploratory factor analysis (EFA), but also a confirmatory factor analysis (CFA) in Japanese community-dwelling elderly. In addition, the effects of age and gender on the performance of the FAI domains obtained from the above factor analyses are also discussed.

\section{Methods}

\section{Subjects and questionnaire}

The study subjects were 1,323 community older residents (375 males [28.3\%], 798 females [60.3\%], 150 gender-missing; the mean age; 74.5 years [S.D. $=6.95]$.) in Hojo City, Ehime prefecture, who were selected from all residents aged 65 years and over $(n=5,916)$ using a systematic random sampling technique. The survey was conducted by the 10 staff of health care centers in Hojo City. They distributed the self-report questionnaires to each subject who agreed to participate, and then collected them in health care centers. The survey contents included demographic information; gender, age, and IADL. The FAI was included in the questionnaires to provide ratings of IADL. The FAI originally comprised 22 items such as preparing meals, shopping, and gardening, but only 15 items selected by Wade et al. (1985), have generally been used. Each item is scored on a four-point scale (0-3), and the scores are summed, giving values between 0 (inactive) and 45 (highly active).

Respondents whose gender was missing or omitting the FAI items were excluded. Due to the missing data for these items, only 696 respondents (290 males [41.7\%], 406 females [58.3\%]; the mean age; 73.5 years [S.D. = 
6.92].) were finally employed for statistical analyses.

The study protocol was approved by the ethical committee of the Hojo City and all the subjects gave informed consent.

\section{Analytic strategies}

In order to examine the factor structure of FAI of 696 subjects, we performed EFA using the exploratory sample (Han et al. 2004, 2005). We estimated a factor solution using M-plus program's weighted least squares parameter estimates and a diagonal weight matrix with robust standard errors and mean- and variance-adjusted chi-square test statistic (WLSMV) (Muthén and Muthén 1998). The number of factors retained was determined according to the theoretical hypothesis, Kaiser's eigenvalue greater-than-1-rule (Kaiser 1960). Interpretation and labeling of retained factors were performed by relying on factor loadings $>0.3$ in PROMAX pattern matrix.

In order to evaluate the robustness of the above factor structure, CFA was performed using a structural equation modeling program AMOS version 4.0 (Arbuckle and Wothke 1999), which is commonly used to perform such analyses (Han et al. 2004, 2005). CFA has several important features over an EFA. CFA enables researchers to systematically test a hypothesis about the internal structure of a psychometric scale by assessing whether the hypothesized model could be fitted to the data (Bryant et al. 1999). In this analysis, a second-order factor model was tested, which represented an underlying construct IADL as a second-order factor dominated by the several first-order factors from the EFA. Furthermore, we used structural equation modeling (SEM) in order to examine the influences of age and gender on the FAI constructs obtained from the above factor analyses.

In the above mentioned CFA and SEM, model fitness to the data was assessed with the chi-square value $\left(\chi^{2}\right)$, Goodness of Fit Index (GFI), Adjusted Goodness of Fit index (AGFI), Comparative Fit Index (CFI), and Root Mean Square Error of Approximation (RMSEA). GFI and AGFI range between 0 and 1, with higher values indicating better fit, and 0.90 generally considered the minimum acceptable level of fit (Bryant et al. 1999). Although a CFI value of 0.90 has served as the lower limit cut-point of an acceptable fit (Bryne 1994; Bryant et al. 1999), a RMSEA value of 0.08 or lower indicates an adequate fit (Bryant 1999). If the model provided inadequate fit indices, we examine the modification indices (MI) (Arbuckle and Wothke 1999) for each parameter in order to improve the model fit. The MI estimates the reduction in chi-square that would result if a particular fixed parameter were free to be estimated in the model as well as the expected change in the value of the particular parameter, given its inclusion in the model. When these modifications are plausible, improve the fit of the model and are well-grounded in preexisting theory, the researcher often frees additional factor loadings or allows for correlated measurement errors in the model.

Significance of parameter estimates (loading paths) were assessed with the values of non-standardized coefficients divided by standardized error (corresponding to $t$ values). $t$ values over $1.96(p<0.05)$ can be judged as statistically significant. Model fit and loading path coefficients were calculated with a maximum likelihood (ML) estimator (Arbuckle and Wothke 1999).

\section{Results}

\section{Study sample}

Respondent characteristics are summarized in Table 1. The sample included 290 males $(41.7 \%)$ and 406 females $(58.3 \%)$. The mean age for all subjects was 73.5 years (s.D. $=6.92$, range 65 - 98). The subjects were aged 65 to 74 (63.4\%), while 255 subjects $(36.6 \%)$ were aged over 75 . The mean age of the male subjects was 73.2 years (s.D. $=6.60)$ and that of the female subjects was 73.7 years $($ S.D. $=7.14)$. There was no significant difference in age between the male and female subjects.

Table 2 shows the distribution of each item for the FAI. The percentage of "never" indicated that the most difficult item was "X15: Gainful work" $(69.7 \%)$, the second most difficult "X14: Reading books" (63.4\%) and then "X13: House/ car maintenance" $(56.3 \%)$. On the other hand, focusing on the "Most days" or "At least once a week" responses, the easiest items were "X8: Walking outdoors" (59.6\%), "X4: Light housework" (55.5\%), and "X6: Local shopping" $(54.6 \%)$.

TABLE 1. Respondent characteristics $(n=696)$.

\begin{tabular}{cccc}
\hline & Male & Female & Total \\
\hline$n(\%)$ & $290(41.7)$ & $406(58.3)$ & $696(100)$ \\
age (s.D.) & $73.2(6.60)$ & $73.7(7.14)$ & $73.5(6.92)$ \\
\hline
\end{tabular}


TABLE 2. Distribution of the responses on the FAI items $(n=696)$.

\begin{tabular}{|c|c|c|c|c|c|c|c|c|c|}
\hline \multirow{2}{*}{ Item } & & \multicolumn{8}{|c|}{ Code } \\
\hline & & \multicolumn{2}{|r|}{0} & \multicolumn{2}{|r|}{1} & \multicolumn{2}{|r|}{2} & \multicolumn{2}{|r|}{3} \\
\hline X 1 & Preparing main meals & 321 & $(46.1)$ & 22 & $(3.2)$ & 59 & $(8.5)$ & 294 & $(42.2)$ \\
\hline X 2 & Washing up & 218 & (31.3) & 36 & (5.2) & 75 & $(10.8)$ & 367 & $(52.7)$ \\
\hline X 3 & Washing clothes & 260 & $(37.4)$ & 40 & $(5.7)$ & 81 & $(11.6)$ & 315 & $(45.3)$ \\
\hline X 4 & Light housework & 188 & $(27.0)$ & 59 & (8.5) & 63 & $(9.1)$ & 386 & $(55.5)$ \\
\hline X 5 & Heavy housework & 210 & $(30.2)$ & 67 & (9.6) & 51 & (7.3) & 368 & (52.9) \\
\hline X 6 & Local shopping & 231 & $(33.2)$ & 34 & (4.9) & 51 & (7.3) & 380 & (54.6) \\
\hline X 7 & Social outings & 336 & $(4.9)$ & 133 & (19.1) & 94 & (13.5) & 133 & (19.1) \\
\hline X 8 & Walking outside $>15$ minutes & 210 & (7.3) & 29 & $(4.2)$ & 42 & $(6.0)$ & 415 & $(59.6)$ \\
\hline X 9 & Actively Pursuing hobby & 268 & $(54.6)$ & 42 & $(6.0)$ & 58 & $(8.3)$ & 328 & $(47.1)$ \\
\hline $\mathrm{X} 10$ & Driving car/bus travel & 206 & $(29.6)$ & 77 & $(11.1)$ & 108 & $(15.5)$ & 305 & $(43.8)$ \\
\hline $\mathrm{X} 11$ & Outings/car rides & 329 & $(47.3)$ & 189 & $(27.2)$ & 78 & $(11.2)$ & 100 & (14.4) \\
\hline $\mathrm{X} 12$ & Gardening & 128 & $(18.4)$ & 255 & $(36.6)$ & 156 & $(22.4)$ & 157 & (22.6) \\
\hline $\mathrm{X} 13$ & Household /car maintenance & 392 & $(56.3)$ & 146 & $(21.0)$ & 76 & $(10.9)$ & 82 & $(11.8)$ \\
\hline $\mathrm{X} 14$ & Reading books & 441 & $(63.4)$ & 115 & $(16.5)$ & 68 & $(9.8)$ & 72 & $(10.3)$ \\
\hline $\mathrm{X} 15$ & Gainful work & 485 & $(69.7)$ & 30 & $(4.3)$ & 66 & $(9.5)$ & 115 & (16.5) \\
\hline & $\begin{array}{l}n(\%) . \\
\text { Item } 1,2: 0=\text { Never, } 1=\text { Under } \\
\text { Item 3-10: } 0=\text { Never, } 1=1-2 \text { tin } \\
\text { Item 11: } 0=\text { Never, } 1=1-2 \text { time } \\
\text { Item 12, } 13: 0=\text { Never, } 1=\text { Ligh } \\
\text { Item 14: } 0=\text { None, } 1=1 \text { in } 6 \text { mo } \\
\text { Item } 15: 0=\text { None, } 1=\text { Up to } 10\end{array}$ & $\begin{array}{r}3 \mathrm{mo} \\
5 \mathrm{mont} \\
= \\
\text { Mod } \\
2=\mathrm{L}\end{array}$ & $\begin{array}{l}\text { ly, } 2=1 \\
\text { nths, } 2= \\
\text { hs, } 2=3 \\
\text { erate, } 3 \\
\text { ess than } \\
2=10-3\end{array}$ & $\begin{array}{l}12 \text { tim } \\
\text { times } \\
1 \text { nece } \\
\text { fortni }\end{array}$ & $\begin{array}{l}\text { week, } 3 \\
\text { es in } 3 \\
\text { in } 3 \text { mo } \\
\text { ssary. } \\
\text { ght, } 3= \\
\text { k, } 3=\mathrm{O}\end{array}$ & $\begin{array}{l}\text { Most } \\
\text { ths, } 3 \\
, 3= \\
\text { r } 1 \text { a }\end{array}$ & $\begin{array}{l}\text { ays. } \\
=\text { at least } \\
\text { it least } \mathrm{x} \\
\text { ortnight } \\
\text { week. }\end{array}$ & & \\
\hline
\end{tabular}

\section{Exploratory factor analysis}

The EFA solutions with oblique rotation (PROMAX) obtained using the maximum likelihood (ML) estimator were examined. The following Eigen values emerged: 4.87, 3.12, 1.04, $0.83,0.81,0.70,0.64,0.57,0.53,0.46,0.40,0.38$, $0.22,0.19$, and 0.17 . The Kaiser's eigenvaluegreater-than-1-rule suggested a three-factor solution. The first latent root accounted for about $29.5 \%$ of the variance of the 15 variables of FAI, and the subsequent two latent roots accounted for $17.7 \%$ and $2.4 \%$ of the variance, respectively. Items 1, 2, 3 and 6 had positive loadings on the first factor, whereas items $7,8,9,10,11,12,13$, 14 and 15 had positive and moderate loadings on the second factor. The third factor had loadings with only two items (Item 4 and 5). Since the Kaiser's rule is known to overestimate the number of latent factors (Zwick and Velicer 1986) and the variance of the third factor was trivial, we ultimately retained the two-factor solution.

The factor matrix is illustrated in Table 3 . In the two-factor solution, items 1, 2, 3, 4, 5, and 6 had large $(\lambda=0.92)$ and at least moderate $(\lambda=$ $0.67)$ loadings on the first factors. The first factor was interpreted as the domestic chores factor. Items $7,8,9,10,11,12,13,14$, and 15 had moderate $(\lambda=0.68)$ and mild $(\lambda=0.37)$ loadings with the second factor. The second factor was labeled the work and leisure factor. The first latent root accounted for about $29.1 \%$ of the variance, and the second latent roots accounted for $17.7 \%$ of the variance. This accounted for $46.8 \%$ of the total variance. The factor correlation matrix demonstrated a weak association between domestic chores and outdoor work and leisure factor $(r=$ $0.16)$. 
TABLE 3. Two-factor solution to exploratory factor analysis of the FAI item $(n=696)$.

\begin{tabular}{llccc}
\hline \multirow{2}{*}{ Item } & & Factor 1 & & Factor 2 \\
\cline { 3 - 3 } X 3 & Washing clothes & Domestic chores & & Work and leisure \\
\hline X 2 & Washing up & $\mathbf{0 . 9 2}$ & -0.12 \\
X 1 & Preparing meals & $\mathbf{0 . 9 0}$ & -0.12 \\
X 4 & Light housework & $\mathbf{0 . 8 5}$ & -0.07 \\
X 5 & Heavy housework & $\mathbf{0 . 8 2}$ & -0.01 \\
X 6 & Local shopping & $\mathbf{0 . 7 2}$ & 0.14 \\
X10 & Driving/bus travel & $\mathbf{0 . 6 7}$ & 0.24 \\
X11 & Outings/car rides & -0.05 & $\mathbf{0 . 6 8}$ \\
X13 & House/car maintanance & 0.02 & $\mathbf{0 . 6 4}$ \\
X12 & Gardening & -0.25 & $\mathbf{0 . 6 4}$ \\
X 9 & Pursuing hobby & -0.03 & $\mathbf{0 . 6 3}$ \\
X 7 & Social outings & 0.24 & $\mathbf{0 . 5 4}$ \\
X 8 & Walking outdoors & 0.13 & $\mathbf{0 . 4 6}$ \\
X14 & Reading books & 0.28 & $\mathbf{0 . 4 1}$ \\
X15 & Gainful work & 0.11 & $\mathbf{0 . 3 8}$ \\
& & -0.19 & $\mathbf{0 . 3 7}$
\end{tabular}

Factor correlation matrix

Domestic chores $\quad 1.00$

$\begin{array}{lll}\text { Work and leisure } & 0.16 & 1.00\end{array}$

Variance explained (\%)

29.1

17.7

Numbers in bold typeface represent factor loadings $>0.35$.

\section{Confirmatory factor analysis}

A confirmatory factor analysis was conducted in order to assess the extent to which FAI factor structure fit to the present data. In this analysis, a second-order factor model was constructed, which showed that the second-order latent factor IADL was a dominant concept in the two firstorder factors obtained from the exploratory factor analysis. Domestic chores factor had five indicators (item 1, 2, 3, 4, 5 and 6). The outdoor work and leisure factor had eight indicators (item 7, 8, 9, $10,11,12,13,14$ and 15). As a result of the CFA, modifications were required for an adequately fitting model, because the model provided poor fit to the data $\left(\chi^{2}[\mathrm{df}]=857.69[89], \mathrm{GFI}=0.85, \mathrm{CFI}\right.$ $=0.84$, RMSEA $=0.11$.

According to the model fit index $(\mathrm{MI}=$
149.14), the improvement in fit was substantial if the error terms of item 4 and 5 of the domestic chores subscale were allowed to covary. The item wordings of these items are similar, and it is plausible that their error terms are correlated. Then, the model fit was moderately improved $\left(\chi^{2}[\mathrm{df}]=\right.$ 694.01[88], GFI $=0.87, \mathrm{CFI}=0.87, \mathrm{RMSEA}=$ $0.10)$. Furthermore, we allowed the error terms of item 12 and 13 of the outdoor subscale to covary based on the value of the modification index (MI $=83.34)$. These items shared a common response category, and, therefore, it is plausible that their error terms are correlated. The modified model demonstrated further improvement in model fit above the baseline model $\left(\chi^{2}\right.$ [df] $=598.41$ [87], $\mathrm{GFI}=0.89, \mathrm{CFI}=0.89$, RMSEA $=0.09)$. After the two modifications, the fit indices were 


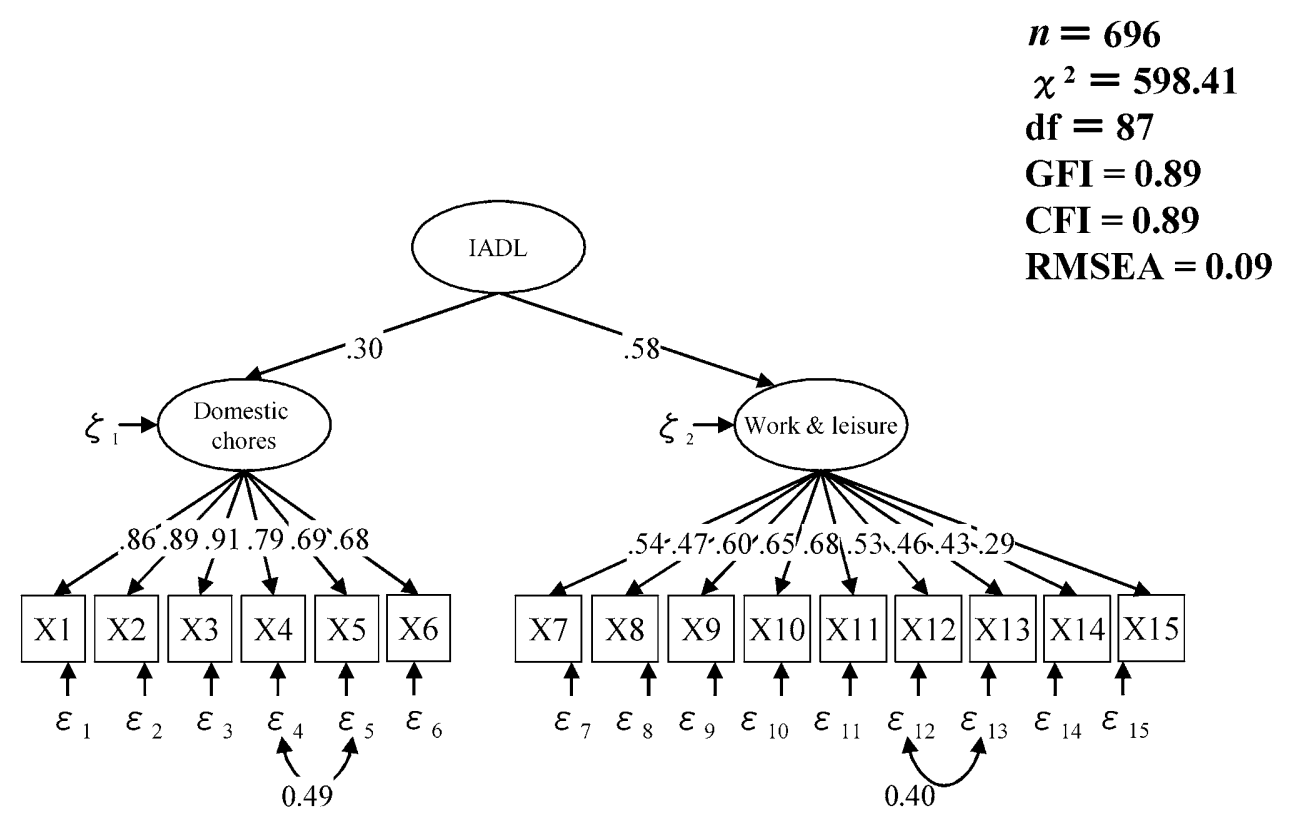

Fig. 1. Final model of the Frenchay Activities Index structure.

The model yielded further improved model fit: $\chi^{2}(\mathrm{df})=598.41(87), \mathrm{GFI}=0.89, \mathrm{CFI}=0.89$, RMSEA $=0.09$. The range of path coefficients in the model was from 0.29 to 0.91 . GFI, Goodness of fit; CFI, Comparative fit index; RMSEA, Root mean square error of approximation.

approximately satisfactory. The final FAI model is displayed in Fig. 1. The model revealed a number of significant paths between latent factors and the indicators. The range of path coefficients was from 0.29 to 0.91 .

\section{Effects of age and gender on the FAI factors}

We performed a SEM in order to examine the age and gender differences in performance on the two FAI domains. The results are shown in Fig. 2. The model fit the data reasonably well $\left(\chi^{2}[\mathrm{df}]=722.25[113], \mathrm{GFI}=0.88, \mathrm{CFI}=0.89\right.$, RMSEA $=0.09)$. All standardized path coefficients were then found to be statistically significant at the $p=0.05$ level. As expected, age and gender differences in performance on domestic chores and outdoor work and leisure were found. Younger age was significantly related to increased performance both on the domestic chores and the work and leisure ( -0.22 and -0.47 , respectively). In addition, relative to females, males had a lower performance on the domestic chores (0.74) and a greater performance on the work and leisure $(-0.19)$.

\section{Discussion}

This study mainly aimed to examine the factor structure of IADL measured by the FAI which was originally developed for stroke patients, using a sample of Japanese community older residents.

In the present study, first, we demonstrated that the FAI had a two-factor structure consisting of domestic chores and work and leisure and the factors had five and eight indicators, respectively. Next, we demonstrated that the FAI using a sample of Japanese community older residents is a second-order factor model consisting of a secondorder factor IADL and two first-order factors, domestic chores and work and leisure, provided somewhat poor fit indices. Moreover, we found that age and gender differences in performance on domestic chores and work and leisure.

Earlier factor analytic studies on the FAI were conducted using a sample of stroke patients and community dwelling elderly and found that the number of factors ranged from two to four. Wade et al. (1985) found a three-factor solution 


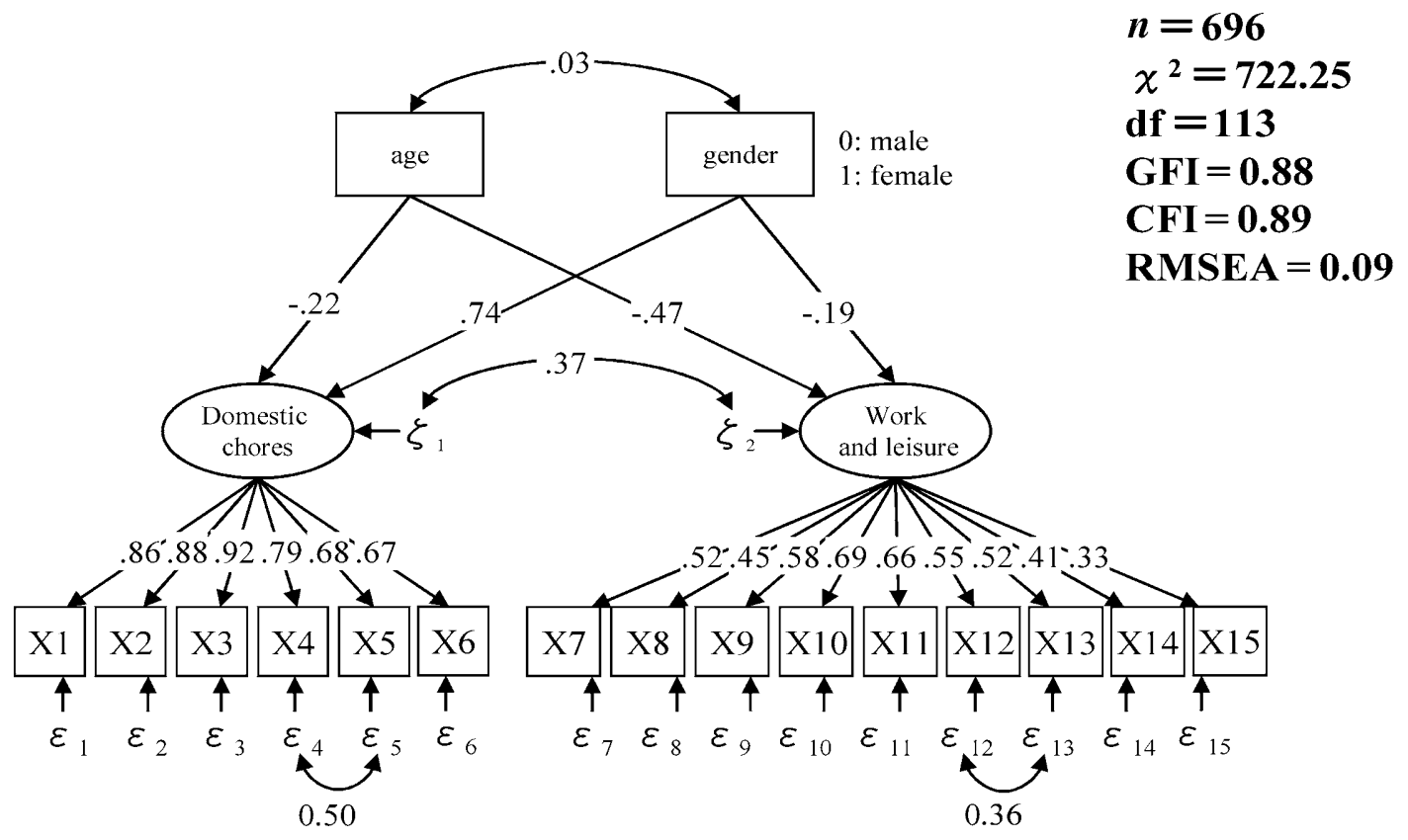

Fig. 2. Effects of age and gender on FAI factors.

The model fit the data reasonably well: $\chi^{2}(\mathrm{df})=722.25(113)$, GFI $=0.88, \mathrm{CFI}=0.89$, RMSEA $=$ 0.09. All standardized path coefficients were then found to be statistically significant $(p=0.05)$. Younger age was significantly related to increased performance both on the domestic chores and the outdoor work and leisure $(-0.22$ and -0.47 , respectively). In addition, relative to females, males had a lower performance on the domestic chores (0.74) and a greater performance on the outdoor work and leisure $(-0.19)$. GFI, Goodness of fit; CFI, Comparative fit index; RMSEA, Root mean square error of approximation.

from the result of factor analysis using a sample of pre-stroke patients. Kburn et al. (1990) also reported a similar three-factor solution. However, these authors did not interpret and label the resulting factors. Bond et al. (1995) also reported a three factor solution including indoor domestic chores, outdoor social activities and outdoor domestic chores factor, but these factor solutions seemed to be an overestimated result. In contrast, Schuling et al. (1993) reported a two-factor solution indicating domestic and outdoor factors, and Sveen et al. (1999) reported a three-factor structure including domestic, outdoor and hobby factors using data from a sample of patients after stroke.

A CFA is a deductive approach and is generally used to test whether some a priori formulated theoretical model adequately explains covariances derived from the data, whereas EFA is an inductive approach and provides a hypothesis for the relationships between a set of items included in a scale and constructs that a researcher intends to measure (Bryant et al. 1999). Unfortunately, little research has focused on the factor structure of the FAI using a CFA, although a CFA has several important advantages over EFA and enables researchers to test a measurement model of the factor underlying a set of questions. Therefore, use of the EFA and CFA together in a factor analytic study is considered to be preferable and it would provide strong evidence concerning the construct validity of the measurement.

Our EFA result found an easily interpretable two-factor solution similar to that reported by Shuling et al. (1993), although the previously reported factor analyses of the FAI are difficult to compare with the present study due to differences in the sample size and composition.

The CFA result indicated that the secondorder factor model consisting of a second-order 
factor IADL and two first-order factors provided somewhat poor fit indices. After two post hoc model modifications, the fit indices were approximately satisfactory. All factor path loadings were medium and large. This supports the EFA result and indicates that the FAI satisfies the assumption of construct validity. However, the factor path loading for the reading item from the work and leisure construct was slightly weak $(\lambda=0.29)$. As Schuling et al. (1993) recommended, the reading item should be deleted, because the item seems to reflect more complex activities, such as effectance suggested by Lawton and Brody (1969), than those addressed by IADL.

The result by the SEM is plausible, when considering that with age, the risk of decline in mental and/or physical functions, or an increased incidence rate of depression is higher. This result was consistent with those reported in previous studies (Wade et al. 1985; Kburn et al. 1990; Sveen et al. 1999). Prominent gender differences also emerged both on the domestic chores and work and leisure. These results were consistent with those of the earlier studies. Female subjects scored significantly higher on the domestic chores subscale than male subjects, but significantly lower on the work and leisure subscale. These subscales may show separate-gender norms for Japanese populations. Therefore, without controlling for gender, researchers would not understand the functional status in IADL of community dwelling elderly subjects. Furthermore, future research is required to make the subscale equivalent to both male and female, for example, by including both male- and female-specific in-home tasks equally.

One of the limitations of the present study was that the response rate was not high: only 696 of $1,323(52.6 \%)$ elderly completed the questionnaires. As the questionnaires were self-reported, the elderly who had low education or low cognitive functions might be not included in this study. There were previous studies that low cognitive functions were associated with ADL in elderly people (Greiner et al. 1996; Fujita et al. 2005). Moreover, we did not examine the associations between the FAI constructs and other factors, e.g., physical, social, economic or environmental factors. It is known that IADL is a multi-dimensional and complex construct and that different people may react differently to apparently similar levels of physical impairment, depending on their expectations, priorities, goals, social-support networks and so on. Further research therefore is necessary in order to decide the clinical importance of the domestic chores and the work and leisure. These attempts would provide a more sophisticated understanding of the IADL in community-dwelling elderly.

In conclusion, we found that the FAI satisfies the construct validity and has a two-factor structure including domestic chores and work and leisure in a sample of Japanese community-dwelling elderly. This finding provides evidence for the construct validity of the FAI, and provides important and clinically valuable information for understanding the patterns of functional status in the Japanese community-dwelling elderly.

\section{References}

Arbuckle, J.L. \& Wothke, W. (1999) Amos 4.0 User's Guide, SmallWater Corporation, USA.

Bond, M.J., Harris, R.D., Smith, D.S. \& Clark, M.S. (1992) An examination of the factor structure of the Frenchay Activities Index. Disabil. Rehabil., 14, 27-29.

Bond, M.J., Clark, M.S., Smith, D.S. \& Harris, R.D. (1995) Lifestyle activities of the elderly: comparison and determinants. Disabil. Rehabil., 17, 63-69.

Bryant, F.B., Yarnold, P.R. \& Michelson, E.A. (1999) Statistical Methodology: VIII. Using confirmatory factor analysis (CFA) in emergency medicine research. Acad. Emerg. Med., 6, 54-66.

Bryne, B.M. (1994) Testing for the factorial validity, replication, and invariance of a measuring instrument: A paradigmatic application based on the Maslach Burnout Inventory. Multivariate Behav. Res., 29, 289-311.

Fujita, K., Kawagoe, M. \& Eto, F. (2005) A longitudinal study of cognitive function and the relationship between cognitive and activities of daily living in the elderly. Nippon Ronen Igakkai Zasshi, 42, 669-676. (in Japanese)

Greiner, P.A., Snowdon, D.A. \& Schitt, F.A. (1996) The loss of independence in activities of daily living: the role of low normal cognitive function in elderly nuns. Am. J. Public Health, 86, 62-66.

Hamrin, E. (1982) One year after stroke: a follow-up of an experimental study. Scand. J. Rehabil. Med., 14, 111-1116.

Han, C.-W., Yajima, Y., Lee, E.-J., Park, C.-M., Meguro, M., Nakajima, K. \& Kohzuki, M. (2004) Development and construct validation of the Korean competence scale (KCS). Tohoku J. Exp. Med., 203, 331-337.

Han, C.-W., Yajima, Y., Lee, E.-J, Nakajima, K., Meguro, M. \& Kohzuki, M. (2005) Validity and utility of the Craig hospital inventory of environmental factors for Korean commu- 
nity-dwelling elderly with or without stroke. Tohoku J. Exp. Med., 206, 41-49.

Holbrook, M. \& Skilbeck, C.E. (1983) An activities index for use with stroke patients. Age Ageing, 12, 166-170.

Kaiser, H.F. (1960) The application of electronic computers to factor analysis, Educ. Psycholo. Meas., 20, 141-151.

Katz, S., Ford, A.B., Moskowitz, R.W., Jackson, B.A. \& Jaffe, W. (1963) Studies of illness in the aged. The index of ADL: a standard measure of biological and psychological function. JAMA, 185, 914-919.

Kburn, J.C., Smith, P.T. \& Wade, D.T. (1990) Influence of cognitive function on social, domestic, and leisure activities of community-dwelling older people. Int. Disabil. Studies, 12, 169-172.

Khin-Heng, D.C. (1995) Measurement of instrument activities of daily living in stroke. Stroke, 26, 1119-1122.

Lawton, M.P. \& Brody, E.M. (1969) Assessment of older people: self-maintaining and instrumental activities of daily living. Gerontologist, 9, 179-186.

Mahoney, F.I. \& Barthel, D.W. (1965) Functional evaluation: the Barthel Index. Md. State Med. J., 14, 61-65.

Moskowitz, E. \& McCann, C.B. (1957) Classification of disability in chronically ill and ageing, J. Chron. Dis., 5, 342-346.
Muthén, L.K. \& Muthén, B.O. (1998) Mplus User's Guide. Los Angeles, CA, USA.

Nouri, F.M. \& Lincoln, N.B. (1987) An extended activities of daily living scale for stroke patients, Clinic. Rehabil., $\mathbf{1}$, 301-305.

Schuling, J., de Haan, R., Limburg, M. \& Groenier, H. (1993) The Frenchay Activities Index; Assessment of functional status in stroke patients. Stroke, 24, 1173-1177.

Soening, H.A., Anderegg, L., Bergstrom, D., Fonda, M., Steinke, N. \& Ulrich, P. (1965) Numerical scoring of selfcare status of patients. Arch. Phys. Med. Rehabil., 46, 689-697.

Sveen, U., Bautz-Holter, E., Sødring, KM., Wyller, T.B. \& Laake, K. (1999) Association between impairments, selfcare ability and social activities 1 year after stroke, Disabil.Rehabil., 21, 372-377.

Wade, D.T., Legh-smith, J. \& Hewer, R.L. (1985) Social activities after stroke: measurement and natural history using the Frenchay Activities Index. Int. Rehabil. Med., 7, 176-181.

Whiting, S. \& Lincoln, N. (1980) An ADL assessment for stroke patients. Br. J. Occup. Ther., 43, 44-46.

Zwick, W. \& Velicer, W. (1986) Comparison of five rules for determining the number of components to retain. Psychol. Bull., 99, 432-442. 